\title{
Research on the Visual Composition of Public Service Advertising Poster Design
}

\author{
$\mathrm{Xin} \mathrm{Cao}^{1, *}$ \\ ${ }^{1}$ Fuzhou University of International Studies and Trade, Fuzhou, Fujian, China \\ *Corresponding author.Email: 350440307@qq.com
}

\begin{abstract}
In this study, the KJ (Affinity Diagram) method is applied, and the following 72 posters are collected. This study collects 72 public service posters and analyzes them in terms of color aesthetics, layout structure, and creative expressions, as well as the literature. However, most of them share the same visual design aesthetics, and among them, the visual expressions of "color to create visual atmosphere", "composition to highlight the theme", and "using expressive techniques to express the author's creative meaning" are the most important.
\end{abstract}

Keywords: public service announcement, poster design, visual composition, visual design aesthetics

\section{INTRODUCTION}

\section{A. Research background and motivation}

Nowadays, we are in the era of rapid development of information, and the diversification of communication media makes the visual expression develop unprecedentedly. In order to keep up with the times, public service posters need to be designed in creative ways, and consider how to add auxiliary power and unique design language to make public service posters equip with emotional value and connotation. In the new culture-oriented society, public service posters need to focus on the psychological and spiritual needs of the audiences. In order to enhance the application value of public service posters, designers can use creative design techniques as creative means to inject cultural power into the posters, so that the posters can interact with the audiences in a spiritual and emotional way, and thus accept the purpose and design concept conveyed by the posters.

Poster design is the most direct and powerful communication medium that enables the audiences to receive information quickly and is widely used in different areas to create value; the main feature of public service advertisements is that they do not aim at profit and combine business interests with social interests (Zhou Guo, 2012). Quality advertising posters can become a work of art that future generations can learn from. The important role of public service posters is to reflect the reality and keep changing the reality to really achieve social progress and harmonious development (Cui Ying, 2013). In order to help contemporary designers to establish and guide the correct awareness of public service, this study will combine the literature to analyze the visual composition of public service advertising poster design, and present the characteristics of excellent works for designers' reference.

\section{B. Purpose of the study}

- Public service advertising was first appeared in the United States in the early 1940s, and it is also known as public service advertising or public moral advertising (Zhou Guo, 2012), and continues to this day. The 21 st century is the era in which we live, and we have learned from the experience of our predecessors and incorporated the best of it into our wisdom.

- The KJ method was used to validate the classification of the 72 samples, and the results were combined with those from the previous literature to form three main directions for secondary validation.

- The "Experience Summary Method" was used to analyze and summarize the findings from the literature review in order to explore the visual composition characteristics of public service posters and advertisements.

\section{LITERATURE REVIEW}

\section{A. Features of public interest advertising posters}

As a form of advertising, public service posters have a strong ideological and artistic character, which can guide the audiences' consciousness and make people establish the right values, so that the public will take the right action. The significant difference between public service posters and commercial posters is that public service posters are not aimed at economic 
interests, but are artistic expressions of public service; the social nature of public service posters determines their wide coverage and strong spreading ability; and the choice of themes mainly revolves around the current hot issues, which can make the public raise the seriousness of the situation to a certain height and strengthen people's responsibility. In terms of persuasion, public service posters can make a good demonstration to correctly guide and gather positive social energy based on creativity, and use infectious visual language to unite people's hearts or warn the audiences.

Contemporary public service advertising posters are different from the traditional posting of large-print newspapers in the past, in the Internet era of advanced communications, it achieves the effect of publicity through a variety of electronic platforms and media to further promote people's public service activities in the form of advertising poster design high frequency circulation. The strong sense of reality can draw the audiences closer to the poster and enhance their psychological identity. This refers to the fact that when people see the poster, the memory savings area in the brain will automatically filter out and directly reflect the expression of certain emotions that resonate with them.

\section{B. Color aesthetics}

Our eyes on the color is like an instinct, we will not always pay special attention to, but often because of color and sigh, life is so pleasing. Wang Changzheng (2018) said that aesthetics is from the perspective of human emotions, focusing on human emotional feedback, and he believes that color aesthetics can be flexibly integrated into the graphic design, to help people appreciate the process of design works to get the heart of the exchange.

\section{Features of the layout structure}

The layout structure tests the basic skills of the designer, and the scattered focus of the audience is a failure. The layout of the layout tests the basic skills of the designer, the audiences' focus on the rhythm is scattered everywhere is a failure, from the very beginning of the layout of the layout of the designer is the designer of the layout of the logical thinking of the judgment error. Julian Liu (2016) proposed six principles as indispensable for a good layout structure, namely: (1) Grouping, (2) Sense of stability, (3) Unity, (4) Grid, (5) White space, and (6) Repetition. Juliana Liu believes that in our lives, there is an inseparable relationship with design, we consciously categorize items in our lives, such as meat products and vegetables in the kitchen to prevent mutual contamination; grouping in layout design is a way to unify information, the picture follows the logical thinking of the designer, placing items like kinship tissues in a similar location, meaning relevance. A sense of stability is a sense of stability and balance that the public is seeking for. The construction of the grid is not only visually stable and comfortable, but also orderly.

In other words, when designing a painting, we already need to set a style framework and think about what kind of theme atmosphere, color tone, and font, etc. are suitable for application on this painting in order to get a unified style; the reasonable use of white space can make the painting feel visually relaxed and make it easier to highlight the main elements. When using repetition techniques, the grouping of kinship groups can enhance the smoothness of reading.

\section{Creative expression in poster design}

Sun Chung-Ping (2015) proposed three creative expression techniques that are in line with modern avant-garde forms: one is dynamic expression technique, i.e., dynamism caused by behavioral participation or composite function; the second is symbolic expression technique, i.e., hand-drawing or computer technique as the main form; and the third is defective expression technique, i.e., the defect of the shape itself or the defect of the homogeneous composition as the main form of expression.

\section{RESEARCH METHODOLOGY AND DATA ANALYSIS}

\section{A. Process of sample analysis by the KJ method of color aesthetics}

From the 72 PSA poster designs of different nature, the 12 groups of PSA poster designs were ranked from the largest to the smallest numerical value in terms of the numerical scale of common and uncommon colors and the meaning of major colors. We can learn from the sample study that firstly, white has the largest number of base in the sample posters, and a clean white background can easily and directly highlight the theme of public service; secondly, the main color with the smallest number of base is yellow, which represents wealth and lightness, and public service publicity firstly does not have commercial value, and secondly, the purpose of public service is to help the underprivileged, so the yellow atmosphere is used in a small amount for public service. In the poster design, (see "Table I"). 
TABLE I. KJ METHOD COLOR AESTHETIC SAMPLE ANALYSIS

\begin{tabular}{lll}
\hline \multicolumn{1}{c}{ Main Shades } & \multicolumn{1}{c}{ Main Atmosphere } & Number \\
\hline White & Pure, Clean, Neat & 16 \\
Blue & Deep, Sensible, Calm & 13 \\
Black & Death, Evil, Secrets & 11 \\
Blue-grey & Elegant, Calm & 7 \\
Anthracite & Bitter, Cold & 5 \\
Tawny & Warm, Old time & 5 \\
Green & Hope, Life, Peace & 4 \\
Brown & Reliable, Stable, Chaotic, Dirty & 3 \\
Red & Hot, Festive, Bloody & 3 \\
Pink & Sweet, Gentle, Innocent & 2 \\
Black, White & Classic, Subdued, Classy & 2 \\
Yellow & Optimism, Enjoyment, Youth & 1 \\
\hline
\end{tabular}

\section{B. Layout structure conforms to the six principles of analysis}

Judging from the composition of the following images, this PSA poster conforms to the six principles: grouping, stability, uniformity, grid, white space, and repetition; (see "Fig. 1") the first impression is the overall atmosphere, using a pink tone, which represents sweetness and innocence, and the audiences' initial impression has established this poster as a symbol of women. As for repetition and grouping, the fonts and shapes used here are grouped at different levels, and through the orderly repetition of pink, they are stably arranged at the bottom of the screen; the large amount of white space gives the audiences space to breathe and directly projects the focus on the center of the theme, forming a visual experience for the audiences. In the composition of quality poster design, the grid is the backbone of a poster, and the orderly arrangement of the images is the indispensable contribution of the grid.

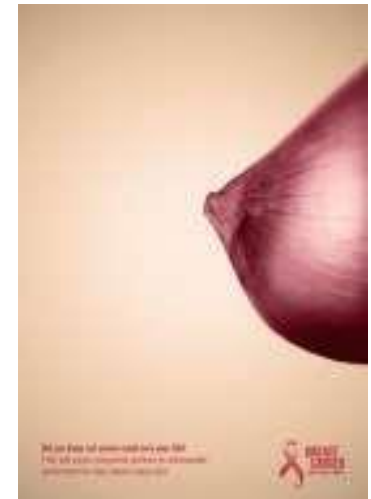

Fig. 1. Poster on caring for women with breast cancer.

\section{Simple analysis of novel approaches}

In recent years, the creative forms of posters have been constantly innovated, and designers are no longer willing to confine themselves to traditional poster design. ("Fig. 2") We can see the dynamic form of this creative poster and people. Every time a shopper puts food into the shopping cart, a hungry child reaches out to beg for food. In fact, how much can the food on hand be worth? Thus, the shopper can feel pity and connect the most normal life of ordinary people with the starving children. Public welfare is a matter of easy work. The poster design of influence is the dynamic behavior participation. According to Sun Zhongping (2015), ("Fig. 3") is a poster design with a dynamic composite function, which can be used as both a poster and a packaging design to eliminate waste and recycling value; ("Fig. 4") is an isomorphic composition, which is formed by the fuzzy jigsaw puzzle of the characters to express the symptoms of Alzheimer's disease, using the elements of the jigsaw puzzle to form the fuzzy characters even though they are put together. ("Fig. 5") In the posters, the audiences are instructed by the designer to feel the eyes closing in the picture to create a dynamic suspense for the "car accident". The audiences will combine the visual impact of the poster with the analogy of the real-life fatigue driving scene to stimulate their understanding of the narrative expression of the metaphorical poster and thus achieve the cognition of the metaphorical content. Therefore, the narrative expression of public service posters can enhance the audiences' understanding of the warning message, and thus achieve the purpose of education and publicity. Poster making by computer is the trend nowadays, ("Fig. 6") uses computer technology to synthesize the picture, the fish is pressed into the body by a plastic straw through the mouth and down the throat to warn people that marine life is suffering and even dying due to the plastic garbage produced by human beings. The escape route in ("Fig. 7") is drawn by hand, the ladder is placed with various objects and hung with blankets, which is wrong, and the red and green color is used to warn people of the importance of the escape route. 


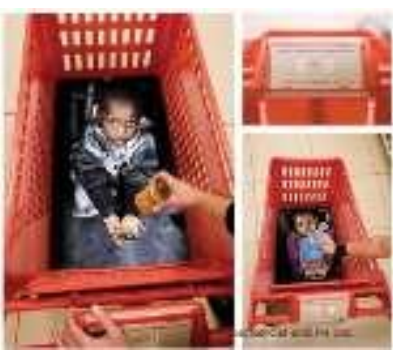

Fig. 2. See how easy feeding the hungry can be?

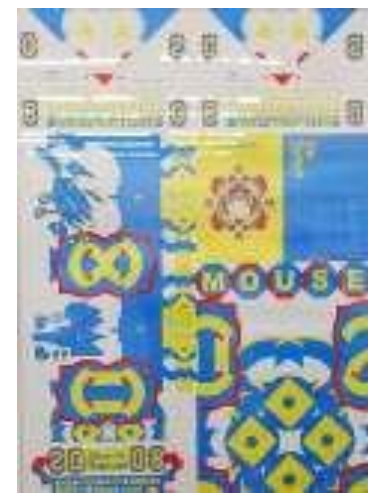

Fig. 3. Rat paper product design.

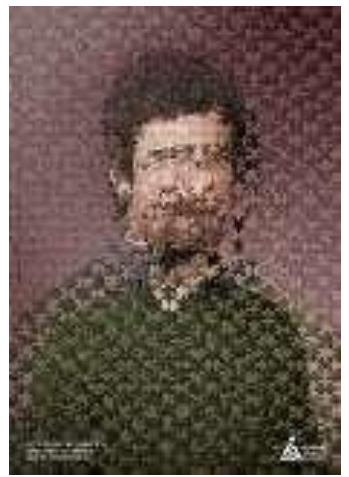

Fig. 4. Alzheimer for Schung initiative.

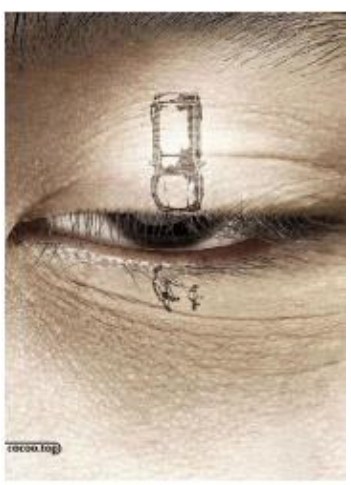

Fig. 5. Driving while fatigued.

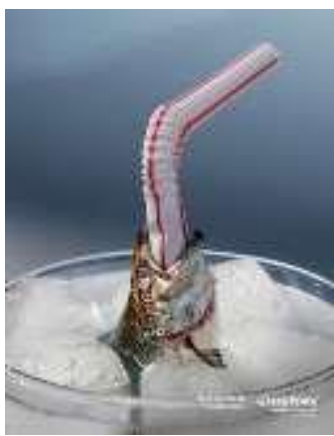

Fig. 6. Don't Suck the Life from Our Oceans.

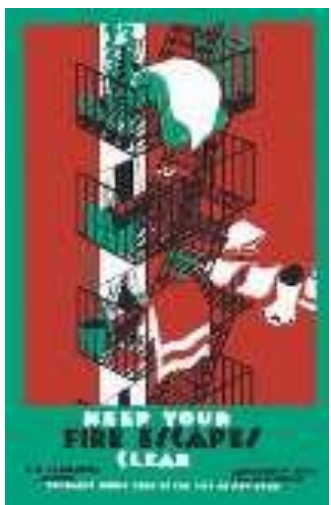

Fig. 7. City of New York.

\section{CONCLUSION}

In summary, based on the application and analysis of visual composition of public service posters, this paper investigates the feasibility and value of creative design techniques in public service posters. First of all, it analyzes the basic meaning and reason for the formation of creative design in public service posters, and further investigates the aesthetic features, design principles and expressions of creative design. Through reading the theoretical studies on creative design and case studies of series of public service posters, the inner emotions and cultural connotations of the creative design approach to public service posters will be revealed to realize the unification of emotions and forms. The combination of creative design techniques and public service posters can make the works transcend their practical functions, and guide the audiences to discover what lies beyond the surface of the works, helping the works to enhance artistic tension and explore the meaning behind art.

In this paper, we focus on the aesthetic features, design principles and expressions of creative design techniques in the report on the high seas, and through a lot of theoretical analysis and case studies, we combine our own design practice to prove that the mutual integration of creative design techniques and public service posters is an inevitable result of the development of the design field. This paper illustrates 
the feasibility of cross-domain reference for art design, and improves the art appreciation, persuasive guidance and communication credibility of the posters. From this, we can see that the application of creative design techniques in the design of public service posters can arouse people's sense of mission, which is the continuation and development of traditional culture, and provides richer expressions for the field of public service poster design. This study shows the importance of visual composition to poster design, and the need for designers to be more professional and sensitive to public service, and also the need to promote cultural progress and establish correct values for the public when the economy is advancing.

\section{References}

[1] Zhou Guo, The weight of public interest advertising. News Sentinel, 2012, 09:108-111.

[2] Cui Ying, The analysis of the design points of public service posters. Aesthetic Education Research, 2013, 05:100.

[3] Li Yingli, An Introduction to the Development of Public Welfare Poster Design under Contemporary Chinese Digital Media Art. Public Art, 2020, 05:58-59.

[4] Chang Zheng, About the Aesthetic Analysis of Color in Graphic Design. Art Education Research, 2018, (03):58-59.

[5] Liu Rui, Exploring the principles of plate structure in layout design. Modern Decoration (Theory), 2016, 04:91-92.

[6] Zhongping Sun, Exploring the individualized expression of modern poster design. Big Stage, 2015, 05:82-83. 